\title{
Analysis of activities undertaken by ward-based clinical pharmacy technicians during patient hospital journey
}

\author{
Ahmed Abuelhana, ${ }^{1,2}$ Linden Ashfield, ${ }_{1}^{3}$ Michael G Scott, ${ }^{3}$ Glenda F Fleming, ${ }^{3}$ \\ Nermin Sabry ${ }_{1}^{4}$ Samar Farid, ${ }^{4}$ Kathryn Burnett (1) ${ }^{1}$
}

'School of Pharmacy and Pharmaceutical Sciences, Faculty of Life and Health Sciences, University of Ulster, Coleraine, UK

${ }^{2}$ Misr University for Science \& Technology, Giza, Egypt ${ }^{3}$ Medicine Optimisation Innovation Center, Northern Health and Social Care Trust, Antrim, UK

${ }^{4}$ Faculty of Pharmacy, Cairo University, Giza, Egypt

\section{Correspondence to}

Prof Kathryn Burnett, School of Pharmacy and Pharmaceutical Sciences, Faculty of Life and Health Sciences, University of Ulster, Coleraine BT52 1SA, UK; k.burnett@ulster.ac.uk

Received 18 April 2019 Revised 16 September 2019 Accepted 30 September 2019 Published Online First 29 October 2019

EAHP Statement 4: Clinical Pharmacy Services.

Check for updates

(C) European Association of Hospital Pharmacists 2021. No commercial re-use. See rights and permissions. Published by BMJ.

To cite: Abuelhana $A$, Ashfield L, Scott MG, et al. Eur J Hosp Pharm

2021:28:313-319.

\begin{abstract}
Introduction Previous studies recognise insufficient time as an obstacle to pharmacists expanding their clinical-based activities and services. For such a reason, the role of well-trained ward-based clinical pharmacy technicians (CPTs) is to work as an integral part of the pharmacy team to achieve the best patient outcomes and medicines optimisation, releasing pharmacist time to complete more complex clinical-related activities.

Objective To demonstrate quantitatively the range and extent of daily activities undertaken by CPTs during a patient's hospital journey.
\end{abstract}

Method A prospective-based study has been designed. All daily working services and activities undertaken by ward-based CPTs within a 450-bed Acute District General hospital were quantitatively collected and documented. Data were collected from five medical, two surgical and one cardiology wards of 30 beds in each over a period of 2 weeks for each ward representing a total of 70 working days (14 weeks, excluding weekends).

Results Results showed the breakdown of seven different ward-based activities throughout a typical working day with the main working load being reviews of the patients' medication charts in order to supply new medicines and refer medicines-related issues to the ward pharmacist, with an average number reviewed of $(23.17 \pm 0.85)$ representing $77.23 \%$ of the total patients in a 30-bed ward. The CPTs' highest workload was on Mondays and Fridays, mainly during the morning working hours (09:00-12:00). Also, statistically significant differences ( $p<0.05$; Kruskal-Wallis test) existed between the workload of the three different ward specialties (medical, surgical and cardiology) in five clinical activities out of seven undertaken by CPT per day.

Conclusion CPTs are completing more than seven different ward pharmacy-related activities which enhance medicines optimisation, medicines management and patient care. They are a valuable resource carrying out many roles which were previously completed by junior pharmacists. Their prioritising of patients for review ensures pharmacists focus their efforts on the most vulnerable patients.

\section{INTRODUCTION}

Clinical pharmacy can be defined as "The area of pharmacy concerned with the science and practice of rational medication use". "Clinical pharmacy services (CPSs) are implemented and provided by a highly trained and skilful team of clinical pharmacists and ward-based clinical pharmacy technicians
(CPTs) to ensure best patient outcomes through optimising all medicines-related needs. ${ }^{2}$ CPSs improve disease and medicines management, health quality and disease prevention through the use of clinical pharmacy staff skills, applied knowledge and gained experiences in addition to the evidencebased practice and continuing education. ${ }^{3}$

Over the past few years, hospital pharmacists' roles and responsibilities have shifted from medication supply into more clinical-related activities including medication history interviews, medicines reconciliation and medicines optimisation where patient safety is the priority within an effective and efficient prescribing and administration environment. ${ }^{4}$ Clinical pharmacy standards were developed in Northern Ireland in 2013, and these were further augmented by publication of the Medicines Optimisation Quality Framework in 2016. ${ }^{56}$ These standards have also been highlighted by the Royal Pharmaceutical Society.

In the UK, pharmacists, pharmacy technicians and pharmacy support staff (dispensing, counter and pharmacy assistants) make up the pharmacy workforce. ${ }^{8}$ They work together with the right skill mix to ensure outstanding CPSs for the best patients' outcomes and cost-effectiveness. ${ }^{9}$ Current pharmacy technician training in Northern Ireland involves completing both a knowledge-based and a competency-based qualification. This is undertaken by part-time study at a local college or online supplemented by work-based training and assessment.

Napier et al stated "Lack of time has been identified as a barrier to pharmacists increasing their clinical activities". ${ }^{10}$ For such a reason, the role of well-trained CPTs is to assist and to support the CPS to achieve the best patient outcomes by medicines optimisation. $^{11}$

This enhancement role of CPTs was highlighted in a review of CPSs in Northern Ireland. It was recognised that pharmacy technicians may be in a position to support the development of CPSs further through involvement in ward-based work. ${ }^{12}$

The Integrated Medicines Management (IMM) project supported this view by promoting the integration of pharmacy technicians into the clinical pharmacy team and training them to perform a limited range of activities to support pharmacists. This released pharmacists' time to deal with more complex medication-related issues. ${ }^{13}$ The IMM was a clinical research project based in Northern Ireland hospitals between 2000 and 2006 to assess and evaluate the clinical and economic impacts of 
increased clinical pharmacy staff involvement throughout the patient's hospital journey from admission to discharge. ${ }^{13}$

At admission, the technician assessed patient's own drugs (PODs) to ensure safety and suitability for use during the patient's stay in hospital. During the inpatient stay, they were responsible for stock management of medicines on the wards including a daily review of the medication prescription chart. The technician also highlighted queries from the chart to their pharmacist. At discharge, they communicated information to primary care health professionals. ${ }^{14}$

Considering how CPTs can work alongside pharmacists in healthcare will promote and aid in developing health systems with the capability of achieving the desirable sustainable goals including optimising patient care and medicines management. ${ }^{15}$

CPSs is a multidisciplinary process achieved by using a variety of competent team members. Currently, the role of CPTs in providing these services is not consistent between different hospitals within Northern Ireland.

\section{Objectives}

The primary objective is to demonstrate quantitatively the range and extent of the daily activities undertaken by ward-based CPTs in terms of integrated medicines management, to enable the best use of the staff resources available based on competencies to complete certain tasks.

\section{METHOD}

This study has been designed as part of a prospective-based CPSs trial, and all daily working services and activities undertaken by ward-based CPTs were quantitatively documented using data collection forms.

On all wards involved in the analysis, the medicines are stored in individual patient bedside lockers. Pharmacy technicians check the medicine prescription chart daily and stock these lockers with medicines. They assess the medicines prescribed and refer a list of standardised issues on to the pharmacist for follow-up. In Northern Ireland, all patients are provided with a 28-day supply of all required medicines at discharge. These are dispensed on each ward using stock from the lockers and final checks will be carried out by CPTs who have a further accredited checking technician qualification.

Initially, a pilot data collection period was carried out in each ward involved in the study for 1 week to standardise data collection by both the CPTs and the principal investigator. This pilot provided reassurance that the same procedures for data collection would be followed in all wards. Data collected during the pilot were not included in the final analysis of the results.

Data were collected from five medical, two surgical and one cardiology wards over a period of 1 to 2 weeks for each ward. This representing a total of 70 days (14 weeks); 15 days from surgical wards, 17 days from cardiology and long-stay wards, and 38 days from medical wards. The pharmacy workforce in each ward comprised one clinical pharmacist and one CPT. The data were collected only during working days from Monday to Friday (weekends were excluded) and between 09:00 and 17:00 every day (night shifts were excluded). The data were collected by the principal investigator, helped by pharmacy staff in each ward. This was achieved by recording all activities completed by CPTs during their typical working day on a data collection form developed by the investigators. CPTs were asked to record their daily activities every day on an hourly basis. Also, the principal investigator contributed on a daily basis to ensure that data were accurately recorded. Data collected included types, numbers and time for each activity The duties were compared from day to day during the working week and hour to hour during the working day and moreover between different ward specialties. Data were collected between April and July 2015.

The period of 24 hours of hospital stay was the minimum duration required to evaluate significant clinical services for any patient within the selected wards.

\section{Study site}

This study was conducted at Antrim Area Hospital, a 450-bed acute general hospital which provides care to a population of almost 436000 people across a geographical area of 1733 square miles.

\section{Statistical analysis}

Analysis of the data was done using SPSS software V.22. Results were reported as mean \pm SEM. Initially, results were tested for normality using Kolmogorov test, then statistical analysis was performed using Kruskal-Wallis test for non-parametric data with pairwise comparison as a post hoc test. Qualitative variables such as percentage were compared using $\chi^{2}$ test.

\section{RESULTS}

Data were collected for 70 working days (from Monday to Friday) within eight different medical and surgical hospital wards with an average number of 30 patients daily in each $(30 \pm 0.51)$.

Results showed that CPTs undertake seven different clinical pharmacy-related activities throughout a typical working day with the main working load focusing on reviewing the patients' medication charts, with an average number reviewed of $23.17 \pm 0.85$ representing $77.23 \%$ of the total patients in the ward (table 1, figure 1). Results revealed also that $15.63 \pm 1.41$ $(67.45 \%)$ of the patients had their medication lockers checked, with $10.84 \pm 0.74(46.78 \%)$ of them requiring medication supply by the CPTs (table 1, figure 1 ). The CPTs' highest workload was on Mondays and Fridays and mainly during the morning working hours (09:00-12:00) (table 1, figure 1).

It has also been shown that the seven clinical pharmacyrelated activities were distributed between morning and afternoon hours. Four activities (patients' medication lockers check, medication lockers supply, patients' medication charts review and referral of patients to the pharmacists for further clinical check) were almost always completed during morning hours (figure 2). On the other hand, three other tasks were mostly completed during the afternoon including assessment of the PODs, labelling and dispensing of medications for discharge, and final checks for the dispensed discharge medicines (figure 2). Patients' medication lockers check and patients' medication charts reviewing were the main work activity of technicians working in all wards (table 1, figure 2).

Results also showed that statistically significant differences ( $p<0.05$; Kruskal-Wallis test) existed between three different ward specialties (medical, surgical and cardiology with longstay wards) in five clinical activities out of seven undertaken by CPT per day (table 2, figure 3 ). However, there were no significant differences ( $p>0.05$; Kruskal-Wallis test) between different weekly days with different ward specialties for all CPT working activities (table 1).

Another service achieved by CPTs was responding to and answering information queries of other healthcare staff within the wards. Results showed that, on average, $1.57 \pm 0.39$ queries were answered daily by CPTs in each ward (figure 4). 


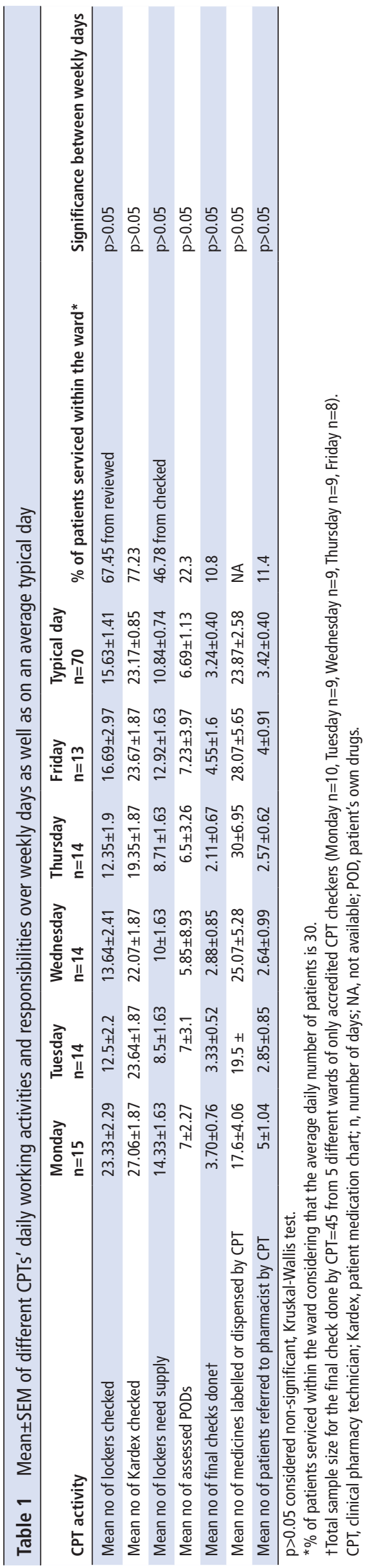

\section{DISCUSSION}

Currently, CPSs are supporting patients during the whole hospital journey (admission, inpatient stay and discharge). Such advanced and standardised services are delivered by competent levels of different pharmacy staff members comprising clinical pharmacists, qualified CPTs and pharmacy assistant technical officers.

CPTs are important partners in the pharmacy workforce with their supporting role allowing pharmacists enough time to focus on more clinical-related activities. ${ }^{16}$ It has been reported that "the expanded role of CPTs improved clinical pharmacy service-delivery, staff satisfaction and patient care". ${ }^{17}$

Throughout this study, all daily working services and activities undertaken by ward-based CPTs were quantitatively collected and documented from different ward specialties including medical, surgical and cardiology wards. All documented activities were analysed and compared between different working days, daily hours and ward specialties.

Seven different clinical pharmacy-related activities were shown to be completed by the ward CPTs working in the hospital. Activities included checking and supply of medicines for patients' medication lockers, reviewing their medication charts, and referring of some patients to the pharmacist for further evaluation and review, as well as dispensing and checking of medications for patients on discharge. These activities are in harmony with the conclusion of Gernant $e t$ al which emphasised the supportive roles of CPTs in patients' medication management. ${ }^{18}$ This wide range of professional services completed by CPTs releases time for the ward clinical pharmacist to carry more therapeutic-related activities, especially for high-risk patients and more complicated cases. The range of activities of CPTs was consistent with the IMM strategy and one-stop dispensing (OSD) policy in the hospital. ${ }^{19} 20$

OSD has been shown to reduce time to complete medication administration rounds, reduce the possibility of medication administration errors and reduce the time of preparation of discharge medicines. It has also been shown to release time for clinical pharmacy staff to carry out more clinical duties, and this was consistent with the findings of Fitzpatrick et $a l^{21}$ and Houlind et al. ${ }^{22}$

The seven clinical pharmacy-related activities completed by CPTs were distributed throughout the day. Four activities based around the Kardex (patient medication chart) review and subsequent supply of medication (patients' medication lockers check, medication charts review, lockers top-up and referral of patients to the pharmacists for further clinical check) were predominately completed during the morning. On the other hand, other tasks mainly based around the discharge process were predominately completed during the afternoon. This included dispensing and final checking of medications for discharges; the final check is almost always completed by an accredited CPT checker. This study identified seven standardised clinical-related activities undertaken by CPTs, responding to Schafheutle et al who stated that the roles of hospital CPTs still need to be well clarified, regulated and standardised. ${ }^{23}$

It was shown also that the workload during the morning was greater than in the afternoon. So, there is the possibility for some extra clinical pharmacy activities to be added to the responsibilities of CPTs during the afternoon. Activities which are more flexible with regards to timing, such as patient counselling on inhalers or direct oral anticoagulants, could be undertaken during this time. 


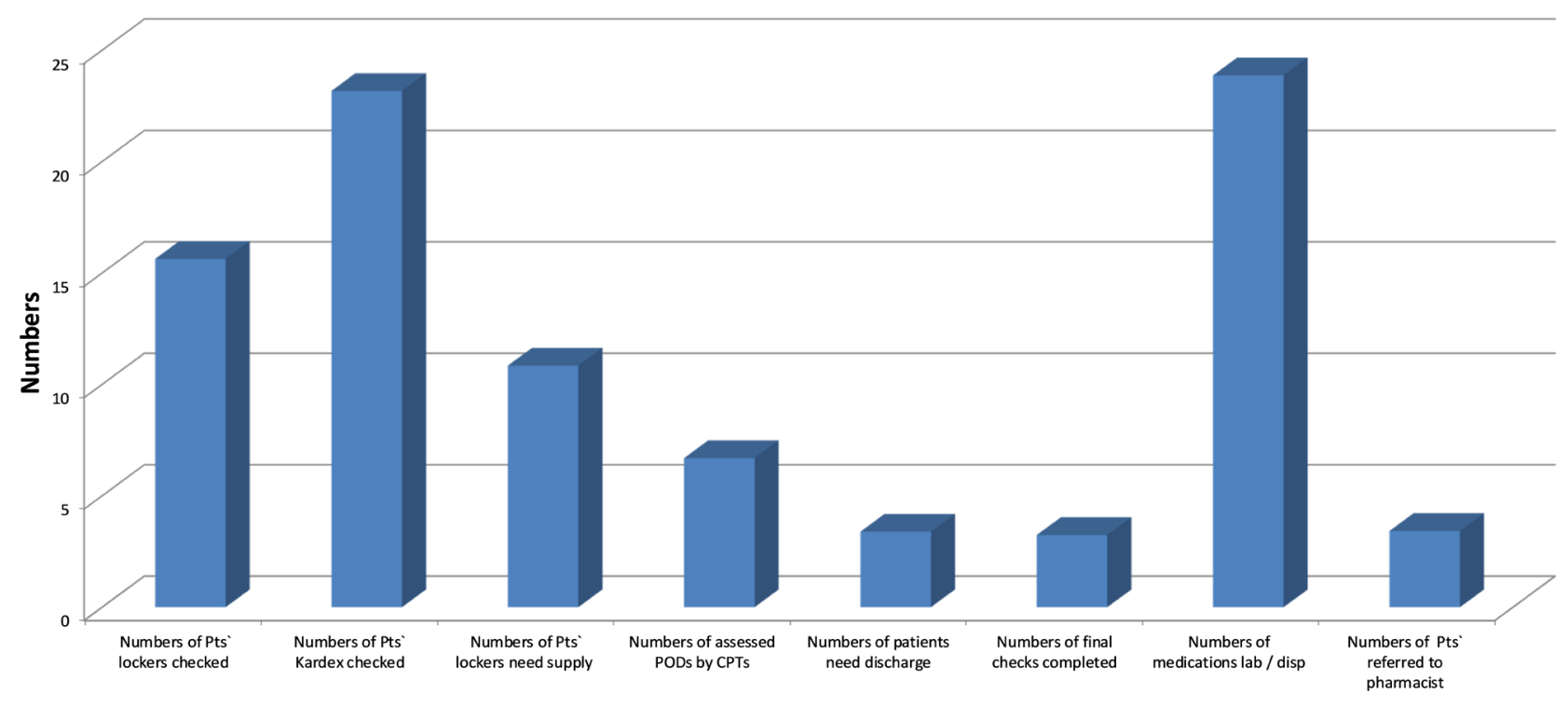

Types of Activities

Figure 1 Average daily working loads and activities completed by ward-based clinical pharmacy technicians (CPTs). POD, patient's own drugs.

As a result of reviewing the medicine charts, CPTs currently refer medication-related issues to the clinical pharmacists according to a standardised medication scheme list. ${ }^{20}$

Significant differences existed in five out of seven activities undertaken by CPTs between the three ward specialties (medical, surgical and cardiology with long-stay wards), and this included numbers of patients' medication lockers check, lockers top-up and referral of patients to the pharmacists for further clinical check in addition to numbers of assessed PODs and final checking of medications for discharges.

Based on findings from other studies, the significant difference may be attributed to the difference in patients' length of the stay between surgical wards and other ward specialties; within surgical wards, patients may stay for a shorter duration than other wards ${ }^{24} 25$ although the daily patient numbers within the wards are similar at around 30 . This was obvious in the significantly increased numbers of medication lockers check, lockers top-up and referral of patients to the pharmacists as well as assessing PODs when compared with other ward specialties.

Also, and according to Nobili et al, within medical and cardiology with long-stay wards, patients almost always have pre-existing comorbidities and consequently are prescribed an increased number of regular medications (polypharmacy), ${ }^{26}$ justifying the imperative need of accurate final checks for patients' discharge prescriptions achieved by accredited CPT checkers. On the other hand, in surgical wards, the discharge prescriptions almost always comprise one, two or three medications, usually none of which is a high-risk medication (medicines which can cause harm or injury to the patient if misused or used incorrectly). For such reasons, the accredited CPT checker is vital in both cardiology and medical wards for

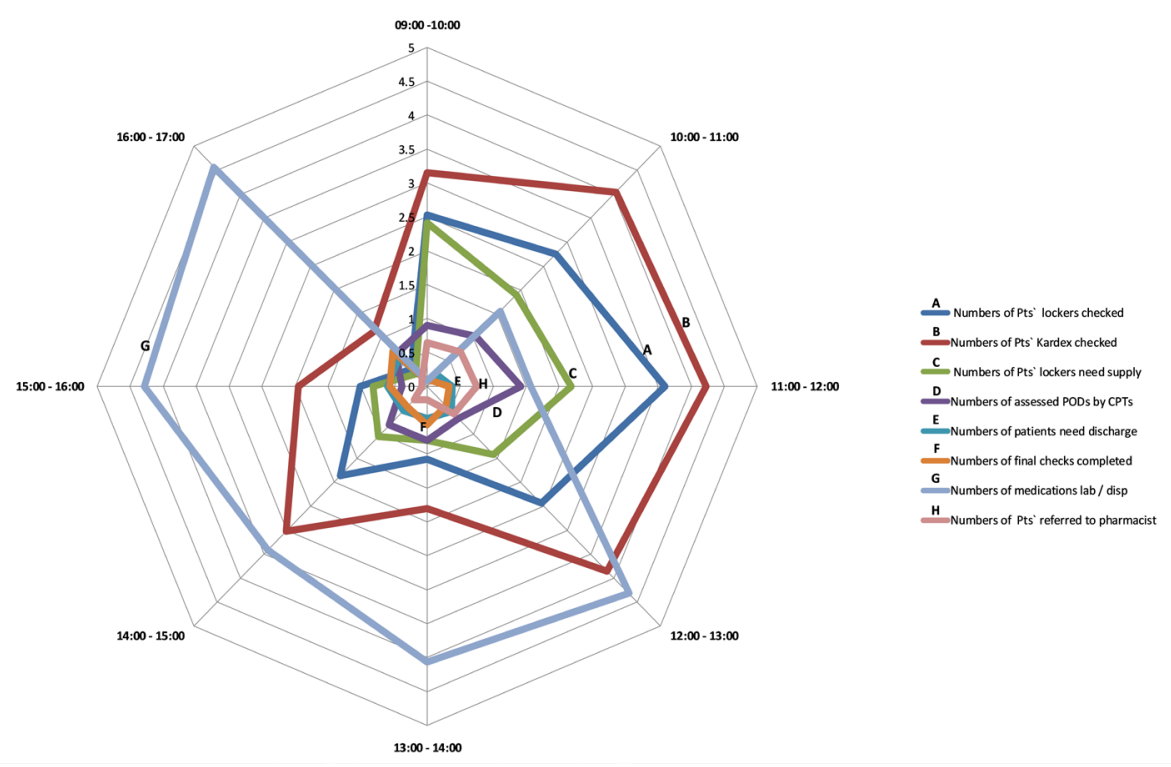

Figure 2 Average distribution of CPTs' activities over typical daily working hours. CPT, clinical pharmacy technician; POD, patient's own drugs. 
Table 2 Mean \pm SEM of different CPTs' daily activities and working load over different ward specialties per day

\begin{tabular}{|c|c|c|c|c|}
\hline CPT activity & Medical wards $n=38$ & Surgical wards $n=15$ & $\begin{array}{l}\text { Cardiology and long-stay } \\
\text { wards } n=17\end{array}$ & Level of significance* \\
\hline Mean no of lockers checked & $15.84 \pm 1.55$ & $21.40 \pm 1.72$ & $10.76 \pm 2.27$ & $p<0.05 \dagger \ddagger$ \\
\hline Mean no of Kardex checked & $22.34 \pm 1.20$ & $22.06 \pm 1.65$ & $26.23 \pm 1.65$ & $p>0.05$ \\
\hline Mean no of lockers need supply & $11.71 \pm 0.96$ & $13.46 \pm 1.74$ & $6.88 \pm 1.12$ & $p<0.05 \dagger \ddagger \S$ \\
\hline Mean no of assessed PODs & $2.73 \pm 0.81$ & $17.53 \pm 4.07$ & $6.05 \pm 2.34$ & $p<0.05+q$ \\
\hline Mean no of final checks done & $3.05 \pm 0.40$ & $0.53 \pm 0.32$ & $3.23 \pm 0.63$ & $\mathrm{p}<0.01+q^{* *}$ \\
\hline $\begin{array}{l}\text { Mean no of medicines labelled or } \\
\text { dispensed by CPT }\end{array}$ & $21.94 \pm 3.79$ & $33.33 \pm 5.66$ & $19.94 \pm 3.63$ & $p>0.05$ \\
\hline $\begin{array}{l}\text { Mean no of patients referred to } \\
\text { pharmacist by CPT }\end{array}$ & $3.50 \pm 0.59$ & $5.46 \pm 0.75$ & $1.47 \pm 0.46$ & $p<0.05 † \ddagger$ \\
\hline
\end{tabular}

*Kruskal-Wallis test for non-parametric data with pairwise comparison as a post hoc test.

$\mathrm{t} \mathrm{p}<0.05$ considered significant.

¥Significant difference between cardiology with long-stay and surgical wards.

$\S$ Significant difference between cardiology with long-stay and medical wards.

ПSignificant difference between medical and surgical wards.

${ }^{* *} p<0.01$ considered highly significant.

CPT, clinical pharmacy technician; POD, patient's own drugs.

accurate checking of the discharge prescriptions for patients with multichronic illnesses.

In terms of workloads over weekly days, there were no significant statistical differences between the three ward specialties in the average numbers of the seven clinical-related activities completed by the ward CPTs.

Answering of medicines' information queries from other healthcare staff in the ward was another activity completed by CPTs within their wards with at least one query every day; this activity was in accordance with the findings of Koehler and Brown ${ }^{15}$ who confirmed that the well-trained, experienced and certified CPTs can independently answer some of the medicines information queries by other healthcare staff. Many different evidenced-based resources were used by CPTs to answer the queries from other healthcare staff in the ward; this included on ward use of British National Formulary, online published local and national guidelines, experience-based competency knowledge and through the help of medicines information centre in the trust. However, most of the answers of CPTs were based on their previous knowledge and experiences.

CPTs have an integral role as part of the CPSs within secondary care. The introduction of electronic prescribing and medicine administration systems in the future will remove some of the tasks undertaken in this study but will provide time to undertake new roles. Currently, pharmacy technicians are not registered in Northern Ireland (unlike colleagues in the rest of the UK). This has to some extent limited the progression to more complex clinical roles.
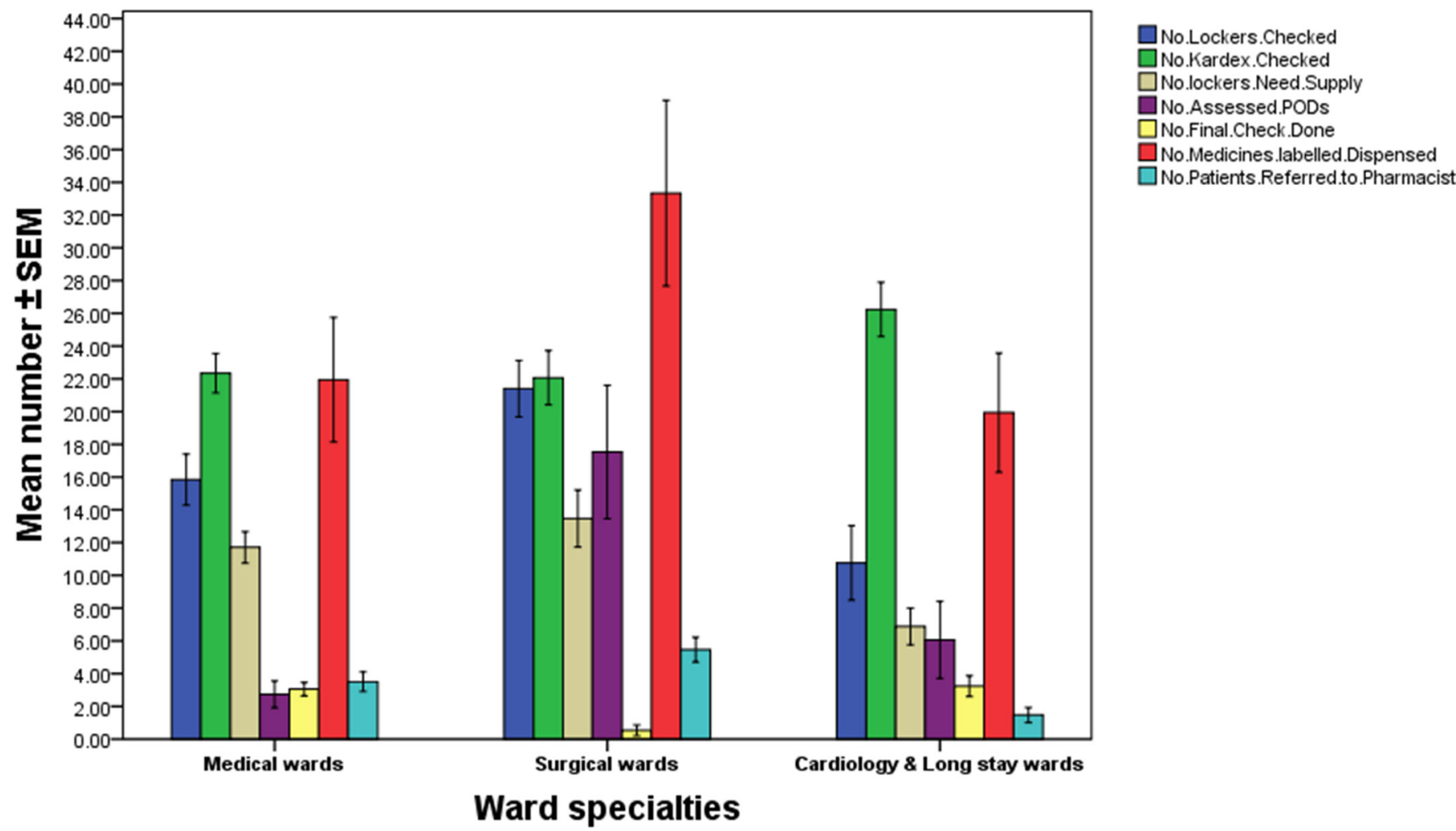

Figure 3 Comparison between workloads of different clinical pharmacy technicians' activities within different ward specialties. 


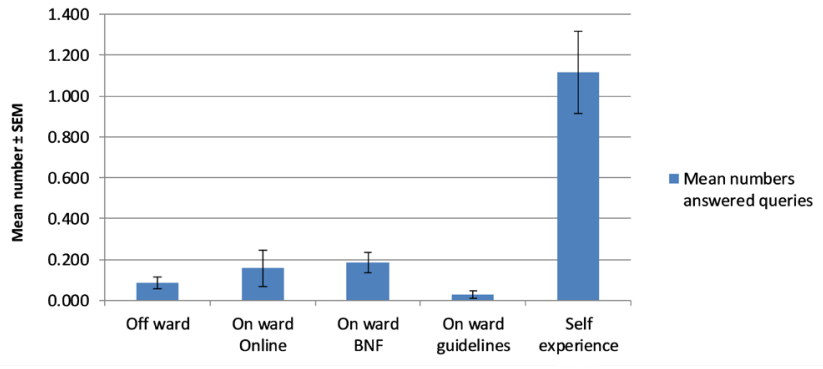

Figure 4 Average numbers of information queries answered by clinical pharmacy technicians per ward every day. BNF, British National Formulary.

Despite this, the clinical part of their role has increased since the study with the introduction of a further accredited course for patient history taking and one for patient counselling currently being developed. This expansion of roles will further increase the skillset of CPTs ensuring optimum CPSs and best patient outcomes.

\section{Limitations}

- Data were mostly collected by the researcher with the support of the working pharmacy workforce and throughout the regular hospital working day, so some data were not possible to be collected such as exact time spent for each task or activity achieved by CPTs in the ward.

- Because data were collected with the support of the working pharmacy workforce, performance bias may be considered as one of the limitations of this study; however, we have piloted the data collection for a week just to ensure the ease of the process and minimise the expected bias.

- This study was conducted only within one hospital, and this may not be accurately reflecting general analysis for CPT activities within other hospitals. Future studies with multicentre testing should be carried out.

\section{CONCLUSION}

CPTs are an integral part of CPSs and their roles can be enhanced to release more time allowing pharmacists to focus on therapeutic issues and use their prescribing skills. CPTs complete more than seven clinical pharmacy-related activities which support the role of medicines optimisation, medicines management and patient care. CPT numbers and bands (grades) as well as competency level such as accredited pharmacy technician checker should

\section{What this paper adds}

What is already known on this subject

- Clinical pharmacy technicians (CPTs) are valuable resources in the clinical pharmacy workforce.

- CPTs can support several clinical-related activities within the hospital; however, there are no clear standards for their activities or scope of their work.

- It is also not clear if services supported by CPTs are provided similarly between different hospitals.

What this study adds

- This study demonstrates quantitatively the range and extent of the daily activities supported by ward-based CPTs.

- This study analyses the current roles of CPTs working in the hospital and provides information to optimise workforce allocation throughout the working day. be considered when allocating CPTs within different clinical specialties of nearly equal patient number.

This study provides an overview of the different CPTs' roles and responsibilities over the working week and during different periods of the working day. This will inform decision-makers on how to make optimum use of available workforce.

This overview will enable the optimum utilisation of the current CPT workforce, providing job satisfaction and career development that meet the needs of service users and carers, thus ensuring the most efficient use of resources to achieve best patients' outcomes.

Further studies are needed to test the impact of development and upgrading of the CPTs to an enhanced clinical role on the clinical pharmacists' responsibilities, time, patients' outcome and cost-effectiveness.

Correction notice This paper has been amended since it was published Online First. The first author's affiliations have been updated.

Funding This research study is part of a larger PhD project partially funded by Misr University for Science and Technology (MUST), Egypt; and supported by Antrim Area Hospital, Northern Health and Social Care Trust (NHSCT), UK.

Competing interests None declared.

Patient consent for publication Not required.

Provenance and peer review Not commissioned; externally peer reviewed.

Data availability statement Data are available on reasonable request.

ORCID iD

Kathryn Burnett http://orcid.org/0000-0001-6451-5271

\section{REFERENCES}

1 American College of Clinical Pharmacy. The definition of clinical pharmacy 2008;28:816-7.

2 Anderson SV, Schumock GT. Evaluation and justification of clinical pharmacy services. Expert Rev Pharmacoecon Outcomes Res 2009;9:539-45.

3 Burke JM, Miller WA, Spencer AP, et al. Clinical pharmacist competencies. Pharmacotherapy 2008;28:806-15

4 Picton C, Wright H. Medicines optimisation: helping patients to make the most of medicines. good practice guidance for healthcare professionals in England. London: Royal Pharmaceutical Society of Great Britain (RPSGB), 2013.

5 Jayne A, Andrew F. How clinical pharmacy practice in Northern Ireland is moving forward. Pharm J 2014.

6 Department of Health, Social Services and Public Safety. Northern Ireland medicines optimisation quality framework, 2016. Available: https://www.health ni.gov.uk/ sites/default/files/consultations/dhssps/medicines-optimisation-quality-framework pdf

7 Martin A. Professional standards for hospital pharmacy services—optimising patient outcomes from medicines. London: Royal Pharmaceutical Society, 2014.

8 Smith Jet al. Securing the future of general practice: new models of primary care. London: Nuffield Trust, 2013.

9 Koehler T, Brown A. Documenting the evolution of the relationship between the pharmacy support workforce and pharmacists to support patient care. Res Social Adm Pharm 2017:13:280-5.

10 Napier P, Norris P, Braund R. Introducing a checking technician allows pharmacists to spend more time on patient-focused activities. Res Social Adm Pharm 2018; 14:382-6.

11 Turner SJ, Lam SS, Leung BK, et al. Establishing the role of a clinical pharmacy technician. J Pharm Prac Res 2005;35:119-21.

12 Department of Health, Social Services and Public Safety. Review of clinical pharmacy services in Northern Ireland, 2001.

13 Scullin C, Scott MG, Hogg A, et al. An innovative approach to integrated medicines management. J Eval Clin Pract 2007;13:781-8.

14 Boughen M, Sutton J, Fenn T, et al. Defining the role of the pharmacy technician and identifying their future role in medicines optimisation. Pharmacy 2017;5.

15 Koehler T, Brown A. A global picture of pharmacy technician and other pharmacy support workforce cadres. Res Social Adm Pharm 2017;13:271-9.

16 Alkhateeb FM, Shields KM, Broedel-Zaugg K, et al. Credentialing of pharmacy technicians in the USA. Int J Pharm Pract 2011;19:219-27.

17 Elliott RA, Perera D, Mouchaileh N, et al. Impact of an expanded ward pharmacy technician role on service-delivery and workforce outcomes in a subacute aged care service. J Pharm Pract Res 2014;44:95-104. 
18 Gernant SA, Nguyen M-O, Siddiqui S, et al. Use of pharmacy technicians in elements of medication therapy management delivery: a systematic review. Res Social Adm Pharm 2018;14:883-90.

19 Scott MG, Scullin C, Hogg A, et al. Integrated medicines management to medicines optimisation in Northern Ireland (2000-2014): a review. Eur J Hosp Pharm 2015:22:222-8.

20 Ashifield $\mathrm{L}$. The impact of standardising referrals by pharmacy technicians on the number of pharmaceutical interventions made by pharmacists for hospital inpatients. Queen's University in Belfast, 2013.

21 Fitzpatrick Ret al. Evaluation of an automated dispensing system in a hospital pharmacy dispensary. Pharm J 2005;274:763-5.
22 Houlind M, McNulty H, Treldal C, et al. One-stop dispensing: hospital costs and patient perspectives on self-management of medication. Pharmacy 2018;6.

23 Schafheutle El, Jee SD, Willis SC. Fitness for purpose of pharmacy technician education and training: the case of Great Britain. Res Social Adm Pharm 2017;13:88-97.

24 Liu Y, Phillips M, Codde J. Factors influencing patients' length of stay. Aust Health Rev 2001:24:63-70

25 Lagoe $\mathrm{R}$, Drapola B, Luziani M, et al. Hospital length of stay reduction: a long-term study. Int J Clin Med 2016:07:530-7.

26 Nobili A, Licata G, Salerno F, et al. Polypharmacy, length of hospital stay, and inhospital mortality among elderly patients in internal medicine wards. The REPOSI study. Eur J Clin Pharmacol 2011;67:507-19. 